\title{
Estimativa do coeficiente de expansão térmica dos acetatos de etila, butila, isoamila, hexila, cis-3-hexenila e trans-2-hexenila
}

\author{
César Augusto CANCIAM* \\ * Mestre em Engenharia Química, professor lotado no Departamento Acadêmico de Engenharia Química da \\ Universidade Tecnológica Federal do Paraná - Câmpus Ponta Grossa, canciam@utfpr.edu.br
}

Recebido em: 20/11/2013 - Aprovado em: 14/09/2014 - Disponibilizado em: 15/12/2014

\begin{abstract}
RESUMO: O conhecimento do coeficiente de expansão térmica de uma substância é importante para avaliar o impacto da dilatação térmica sobre a medição volumétrica. O presente trabalho teve por objetivo estimar o coeficiente de expansão térmica dos acetatos de etila, butila, isoamila, hexila, cis-3-hexenila e trans-2-hexenila. Para tanto, foi realizada uma análise de regressão linear de dados experimentais da densidade de cada éster em função da temperatura. Estes valores foram encontrados em outro trabalho. Os valores do coeficiente de expansão térmica para os ésteres estudados encontram-se entre $6,4995 \times 10^{-4}$ e $10,8554 \times 10^{-4} \mathrm{~K}^{-1}$. Em todos os casos, os coeficientes de correlação foram próximos da unidade, classificando a correlação linear como muito forte.
\end{abstract}

PALAVRAS-CHAVE: Dilatação. Ésteres. Estimativa. Regressão linear. Acetatos.

\section{Estimate of the thermal expansion coefficient of ethyl, butyl, isoamyl, hexyl, cis- 3-hexenyl and trans-2-hexenyl acetates}

ABSTRACT:The knowledge of thermal expansion coefficient of a substance is important to evaluate the impact of thermal expansion of the volumetric measurement. This study aimed to estimate the thermal expansion coefficient of ethyl, butyl, isoamyl, hexyl, cis-3-hexenyl and trans-2-hexenyl acetates. For this purpose, there was a linear regression analysis of experimental data of density of each ester in a function of temperature. These values were found in another study. The thermal expansion coefficients for esters studied are between $6.4995 \times 10^{-4}$ and $10.8554 \times 10^{-4} \mathrm{~K}^{-1}$. In all cases, the correlation coefficients were near to unit, classifying as very strong linear correlation.

KEYWORDS: Dilatation. Esters. Estimate. Linear regression. Acetates

\section{INTRODUÇÃO}

Os ésteres são compostos orgânicos derivados dos ácidos carboxílicos pela substituição da hidroxila por um grupo alquila ou arila. Via de regra, são líquidos; menos densos que a água; com ponto de ebulição normal, devido à ausência de ligações de hidrogênio (CAMPOS et al., 1997; SKORONSKI, 2006).
Segundo Solomons e Fryhle (2012), as moléculas dos ésteres apresentam certa polaridade devido à presença de dois átomos de oxigênio e ao ângulo formado entre as ligações dos átomos no grupo funcional. Essa polaridade é mais acentuada em ésteres de cadeia carbônica pequena (com massa molecular pequena), em que predominam as interações intermoleculares do tipo dipolo permanente-dipolo permanente. À medida que a massa molecular do éster aumenta, a 
polaridade vai se tornando menor e passam a existir interações intermoleculares do tipo dipolo instantâneo-dipolo induzido.

Os ésteres são frequentemente empregados como solventes em reações orgânicas porque formam um meio reacional moderadamente polar e aprótico. Por esse motivo, são utilizados como solventes em diversos setores industriais como petroquímica, alimentos, cosméticos, tintas e vernizes, entre outros (SKORONSKI, 2006).

Um grande número de ésteres é volátil e apresenta odor agradável, sendo que muitos deles são utilizados na indústria de perfumes ou na formulação de aromas empregados nos alimentos (BRUICE, 2006).

A Tabela 1 relaciona os ésteres estudados neste trabalho e seu respectivo aroma característico.

Tabela 1 - Aromas característicos dos ésteres estudados

\begin{tabular}{cc}
\hline Éster & Aroma \\
\hline Acetato de etila & Conhaque \\
Acetato de butila & Maçã verde \\
Acetato de isoamila & Banana \\
Acetato de hexila & Pera \\
Acetato de cis-3-hexenila & Chá verde
\end{tabular}
Acetato de trans-2-hexenila Maracujá Fonte: DJOJOPUTRO e ISMADJI (2005); VIEIRA (2006).

Chung e coautores (1996) comentam que a densidade $(d)$ é uma propriedade da matéria que está intimamente relacionada com a temperatura e a pressão. A densidade é uma das mais fundamentais propriedades de um material e está intrinsicamente relacionada com outras propriedades. Uma dessas propriedades é o coeficiente de expansão térmica $(\beta)$.

O coeficiente de expansão térmica está relacionado com a resposta dos materiais à aplicação de calor. E fornece uma medida da variação da densidade em resposta a uma mudança de temperatura, sob uma condição de pressão constante (INCROPERA e DEWITT, 1998; CALLISTER, 2002).

A Equação 1 relaciona a densidade com a variação da temperatura e o coeficiente de expansão térmica. (CANCIAM, 2005).

$$
\ln \left(\frac{d_{0}}{d}\right)=\beta \cdot\left(T-T_{0}\right)
$$

Em que $T_{0}$ e $T$ correspondem, respectivamente, à temperatura inicial e à temperatura final; e, $d_{0} \mathrm{e} d$ correspondem, respectivamente, às densidades nessas temperaturas

A Equação 1 corresponde a uma função afim. Dessa forma, o gráfico de $\ln \left(\frac{d_{0}}{d}\right)$ versus $\left(T-T_{0}\right)$ fornece uma reta, em que o coeficiente angular corresponde, numericamente, ao coeficiente de expansão térmica (CANCIAM, 2010).

Santos e Vieira (2010) comentam que a partir do conhecimento do coeficiente de expansão térmica é possível estimar outras propriedades físico-químicas e avaliar os 
impactos nos sistemas de medição volumétrica devidos à mudança de temperatura.

O objetivo deste trabalho foi estimar o coeficiente de expansão térmica dos acetatos de etila, butila, isoamila, hexila, cis-3hexenila e trans-2-hexenila. A estimativa do coeficiente de expansão térmica de cada éster utilizou dados experimentais da densidade em função da temperatura encontrados em outro trabalho.

\section{MATERIAIS E MÉTODOS}

A Tabela 2 relaciona os dados experimentais da densidade em função da temperatura para os acetatos de etila, butila e isoamila.

Tabela 2. Efeito da temperatura na densidade dos acetatos de etila, butila e isoamila

\begin{tabular}{cccc}
\hline $\begin{array}{c}\text { Temperatura } \\
(\mathrm{K})\end{array}$ & $\begin{array}{c}\text { Densidade } \\
\text { do acetato } \\
\text { de etila } \\
\left(\mathrm{g} \cdot \mathrm{cm}^{-3}\right)\end{array}$ & $\begin{array}{c}\text { Densidade } \\
\text { do acetato } \\
\text { de butila } \\
\left(\mathrm{g} . \mathrm{cm}^{-3}\right)\end{array}$ & $\begin{array}{c}\text { Densidade } \\
\text { do acetato } \\
\text { de isoamila } \\
\left({\left.\mathrm{g} . \mathrm{cm}^{-3}\right)}^{-3}\right.\end{array}$ \\
\hline 293,15 & 0,90048 & 0,88104 & 0,87824 \\
298,15 & 0,89481 & 0,87645 & 0,87601 \\
303,15 & 0,88714 & 0,87130 & 0,87594 \\
308,15 & 0,88221 & 0,86625 & 0,87310 \\
313,15 & 0,87892 & 0,86184 & 0,87015 \\
318,15 & 0,87451 & 0,85732 & 0,86732 \\
323,15 & 0,87039 & 0,85461 & 0,86431 \\
328,15 & 0,86713 & 0,85078 & 0,86014 \\
333,15 & 0,86472 & 0,84792 & 0,85798 \\
338,15 & 0,86141 & 0,84439 & 0,85405 \\
343,15 & 0,85836 & 0,84177 & 0,85107 \\
\hline
\end{tabular}

Fonte: Djojoputro e Ismadji (2005).

Enquanto que a Tabela 3 relaciona os dados experimentais da densidade em função da temperatura para os acetatos de hexila, cis3-hexenila e trans-2-hexenila.

Os dados indicados nas Tabelas 2 e 3 foram substituídos nas Equações 2, 3, 4, 5, 6, 7, 8 e 9. Essas equações são adaptações do trabalho de Triola (2008) para a análise de regressão linear.

Tabela 3. Efeito da temperatura na densidade dos acetatos de hexila, cis-3-hexenila e trans-2-hexenila

\begin{tabular}{|c|c|c|c|}
\hline $\begin{array}{c}\text { Temperatura } \\
(\mathrm{K})\end{array}$ & $\begin{array}{c}\text { Densidade } \\
\text { do acetato } \\
\text { de hexila } \\
\left(\mathrm{g} . \mathrm{cm}^{-3}\right)\end{array}$ & $\begin{array}{c}\text { Densidade } \\
\text { do acetato } \\
\text { de cis-3- } \\
\text { hexenila } \\
\left(\mathrm{g} \cdot \mathrm{cm}^{-3}\right)\end{array}$ & $\begin{array}{c}\text { Densidade } \\
\text { do acetato } \\
\text { de trans-2- } \\
\text { hexenila } \\
\left(\mathrm{g} . \mathrm{cm}^{-3}\right)\end{array}$ \\
\hline 293,15 & 0,87290 & 0,90082 & 0,89784 \\
\hline 298,15 & 0,86730 & 0,89714 & 0,89432 \\
\hline 303,15 & 0,86260 & 0,89314 & 0,89178 \\
\hline 308,15 & 0,85833 & 0,89028 & 0,88846 \\
\hline 313,15 & 0,85490 & 0,88619 & 0,88435 \\
\hline 318,15 & 0,84884 & 0,88143 & 0,88019 \\
\hline 323,15 & 0,84421 & 0,87789 & 0,87614 \\
\hline 328,15 & 0,83964 & 0,87521 & 0,87281 \\
\hline 333,15 & 0,83515 & 0,87143 & 0,86749 \\
\hline 338,15 & 0,83140 & --- & --- \\
\hline 343,15 & 0,82630 & --- & --- \\
\hline
\end{tabular}

$$
\begin{gathered}
\beta=\left\{\frac{n \cdot a-b}{n \cdot c-h}\right\} \\
R^{2}=\frac{(n \cdot a-b)}{(\sqrt{(n \cdot c-h)} \cdot(\sqrt{(n \cdot e-f)})} \\
a=\left\{\sum\left[\left(T-T_{0}\right) \cdot \ln \left(d_{0} / d\right)\right]\right\} \\
b=\left\{\left[\sum\left(T-T_{0}\right)\right] \cdot\left[\sum \ln \left(d_{0} / d\right)\right]\right\} \\
c=\sum\left(T-T_{0}\right)^{2} \\
e=\sum\left[\ln \left(d_{0} / d\right)\right]^{2} \\
f=\left[\sum \ln \left(d_{0} / d\right)\right]^{2} \\
h=\left[\sum\left(T-T_{0}\right)\right]^{2}
\end{gathered}
$$


Em que, $R^{2}$ corresponde ao coeficiente de correlação e $n$, ao número de dados amostrais emparelhados.

Com base nas Tabelas 2 e 3, $n$ é igual a 11 para os acetatos de etila, butila, isoamila e hexila e igual a 9 para os acetatos de cis-3hexenila e trans-2-hexenila.

Para cada éster, a densidade inicial $\left(d_{0}\right)$ corresponde à densidade na temperatura inicial $\left(T_{0}\right)$ de $293,15 \mathrm{~K}$.

\section{RESULTADOS E DISCUSSÃO}

A Figura 1 ilustra o gráfico de $\ln \left(\frac{d_{0}}{d}\right)$ versus $\left(T-T_{0}\right)$ para os acetatos estudados.

Figura 1: Gráfico de $\ln \left(\frac{d_{0}}{d}\right)$ versus $\left(T-T_{0}\right)$ para os acetatos estudados

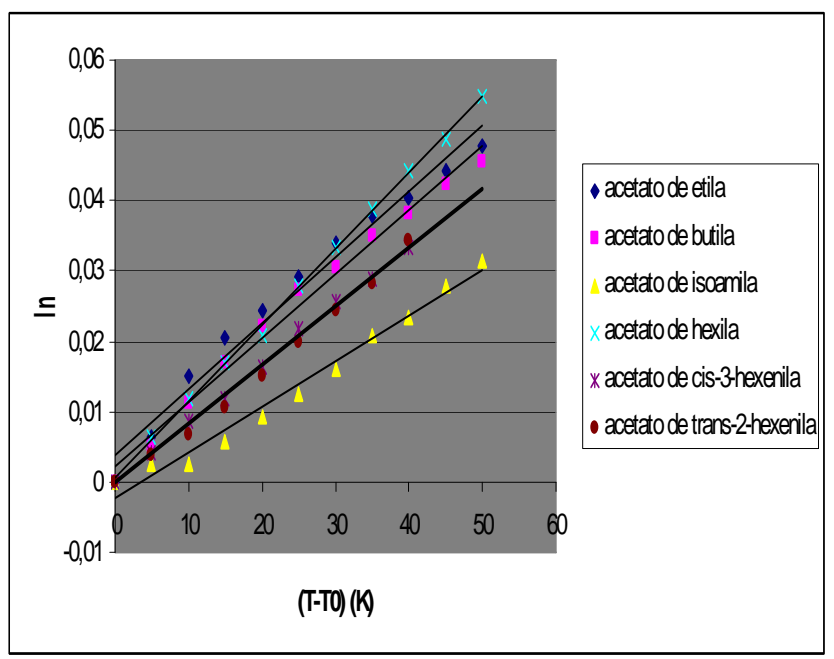

Fonte: Autor.
A Tabela 4 indica os resultados obtidos através das Equações 2 e 3

Tabela 4. Coeficientes de expansão térmica e de correlação dos ésteres estudados

\begin{tabular}{ccc}
\multicolumn{3}{c}{ correlação dos ésteres estudados } \\
\hline Éster & $\beta \cdot 10^{-4}$ & $R^{2}$ \\
& $\left(\mathrm{~K}^{-1}\right)$ & \\
\hline Acetato de etila & 9,3220 & 0,9892 \\
Acetato de butila & 9,1474 & 0,9950 \\
Acetato de isoamila & 6,4995 & 0,9911 \\
Acetato de hexila & 10,8554 & 0,9995 \\
Acetato de cis-3-hexenila & 8,3826 & 0,9990 \\
Acetato de trans-2-hexenila & 8,5109 & 0,9970 \\
\hline
\end{tabular}

Fonte: Autor.

Pode-se observar na Tabela 4, que o coeficiente de expansão térmica dos ésteres estudados neste trabalho varia de 6,4995.10 $0^{-4}$ a $10,8554 \cdot 10^{-4} \mathrm{~K}^{-1}$.

Com relação ao coeficiente de correlação, Triola (2008) comenta que esse coeficiente mede o grau de relacionamento linear entre os valores emparelhados das variáveis dependente e independente. Ou seja, quanto mais próximo for esse coeficiente da unidade, melhor é a qualidade do ajuste da função em relação aos pontos do diagrama de dispersão.

Lira (2004) acrescenta que para valores do coeficiente de correlação maiores ou iguais a 0,90 e menores que 1 , a correlação linear é classificada como muito forte.

Com base na Tabela 4, pode-se verificar que para todos os acetatos estudados, a correlação linear é classificada como muito forte. 
A Figura 2 compara os coeficientes de expansão térmica dos acetatos estudados neste trabalho com os coeficientes de expansão térmica de outros ésteres encontrados na literatura.

Nota-se na Figura 2 que o coeficiente de expansão térmica do acetato de isoamila $\left(6,4995.10^{-4} \mathrm{~K}^{-1}\right) \quad$ encontra-se entre os coeficientes de expansão térmica dos butiratos de etila $\left(6,43 \cdot 10^{-4} \mathrm{~K}^{-1}\right)$ e butila $\left(7,45 \cdot 10^{-4} \mathrm{~K}^{-1}\right)$.

Para o coeficiente de expansão térmica do acetato de cis-3-hexenila $\left(8,3826.10^{-4} \mathrm{~K}^{-1}\right)$, este encontra-se entre os coeficientes de expansão térmica do isovalerato de etila $\left(7,8473.10^{-4} \mathrm{~K}^{-1}\right)$ e o hexanoato de etila $\left(8,4063 \cdot 10^{-4} \mathrm{~K}^{-1}\right)$.

Figura 2: Comparação entre os coeficientes de expansão térmica dos ésteres

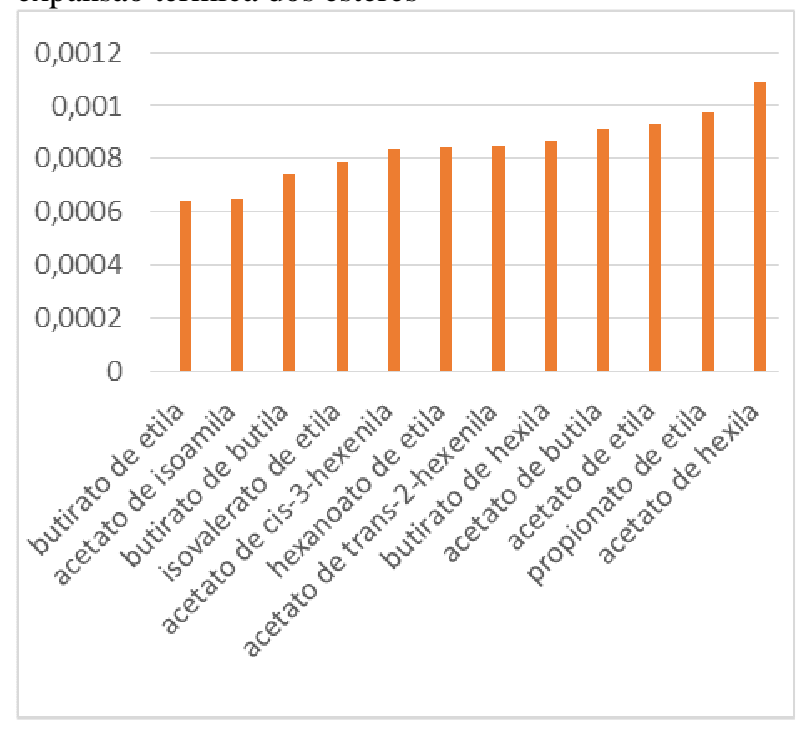

Fonte: Autor.

Ainda na Figura 2, em termos dos coeficientes de expansão térmica, o acetato de trans-2-hexenila $\left(8,5109.10^{-4} \mathrm{~K}^{-1}\right)$ encontra-se entre o hexanoato de etila $\left(8,4063 \cdot 10^{-4} \mathrm{~K}^{-1}\right)$ e o butirato de hexila $\left(8,70.10^{-4} \mathrm{~K}^{-1}\right)$.

Os ésteres acetatos de butila $\left(9,1474.10^{-4} \mathrm{~K}^{-1}\right) \quad$ e etila $\left(9,3220.10^{-4} \mathrm{~K}^{-1}\right)$ encontram-se entre os ésteres butirato de hexila $\left(8,70.10^{-4} \mathrm{~K}^{-1}\right)$ e propionato de etila $\left(9,7736 \cdot 10^{-4} \mathrm{~K}^{-1}\right)$.

Enquanto que, o éster acetato de hexila $\left(10,8554.10^{-4} \mathrm{~K}^{-1}\right)$ apresenta o maior valor para o coeficiente de expansão térmica, superando o éster propionato de etila $\left(9,7736.10^{-4} \mathrm{~K}^{-1}\right)$.

Os coeficientes de expansão térmica dos butiratos de etila, butila e hexila são encontrados no trabalho de Canciam (2012a). Já os coeficientes de expansão térmica dos ésteres isovalerato de etila, hexanoato de etila e propionato de etila são encontrados no trabalho de Canciam (2012b).

Santos e Vieira (2010) comentam que os valores do coeficiente de expansão térmica estão relacionados com a energia de ligação química entre as partículas, de maneira que, materiais em que as ligações químicas são fortes, os coeficientes de expansão térmica são baixos. Isto porque, segundo Padilha (1997), a dilatação térmica está associada à variação assimétrica da energia de ligação com a distância entre as partículas. Ou seja, durante o aquecimento, as partículas do material aumentam a frequência e a amplitude 
da vibração e como as forças de repulsão são maiores que as forças de atração, aumenta-se a distância média entre as partículas.

Assim, os resultados obtidos para os coeficientes de expansão térmica sugerem que no acetato de hexila as forças de atração entre suas moléculas são mais fracas, enquanto que no acetato de isoamila, as forças de atração entre suas moléculas são mais fortes.

Com relação aos impactos da dilatação térmica no sistema de medição, a ordem de aumento no volume dos ésteres estudados varia de 6,50 (acetato de isoamila) a 10,86 litros (acetato de hexila), considerando uma variação de 10K para cada 1000 litros do éster específico.

$\mathrm{O}$ acetato de isoamila, quando comparado com a água $\left(2,07.10^{-4} \mathrm{~K}^{-1}\right)$ (CABRAL e LAGO, 2002), a ordem de aumento no volume equivale a 3,14 vezes a ordem de aumento da água. Para o acetato de hexila, esse valor atinge 5,25 vezes a ordem de aumento de água.

\section{CONCLUSÃO}

A utilização de dados experimentais da densidade em função da temperatura permitiu estimar os coeficientes de expansão térmica dos acetatos de etila, butila, isoamila, hexila, cis-3-hexenila e trans-2-hexenila.
Os valores estimados para os coeficientes de expansão térmica variaram de $6,4995.10^{-4} \mathrm{~K}^{-1}$ (acetato de isoamila) a $10,8554.10^{-4} \mathrm{~K}^{-1}$ (acetato de hexila).

Em comparação a outros ésteres, os valores estimados neste trabalho encontramse próximos.

Em todas as análises de regressão linear, a correlação linear foi classificada como muito forte.

Com base nos resultados obtidos, sugere-se que no acetato de hexila as forças de atração entre suas moléculas são mais fracas, enquanto que no acetato de isoamila, estas são mais fortes.

\section{REFERÊNCIAS}

BRUICE, P. Y. Química Orgânica - volume 2. Pearson Education do Brasil, São Paulo, 2006. 641p.

CABRAL, F.; LAGO, A. Física 2. Harbra, São Paulo, 2002. 516p.

CALLISTER, W. D. Ciência e Engenharia de Materiais: uma introdução. Livros Técnicos e Científicos, Rio de Janeiro, 2002. 450p.

CAMPOS, M. M.; AMARAL, L. F. P.; SEOUD, O. E.; ALVARENGA, M. A.; VICENTINI, G.; YOSHIDA, M.; LOPES FILHO, J. C.; DE' ÁCQUA, A. Fundamentos de Química Orgânica. Edgard Blücher, São Paulo, 1997. 606p.

CANCIAM, C. A. Predição do coeficiente de expansão térmica do óleo de algodão.

Publicatio UEPG - Ciências Exatas e da 
Terra, Ciências Agrárias e Engenharias, v. 11, n. 3, p. 27-31, 2005.

CANCIAM, C. A. Predição do coeficiente de expansão térmica do óleo de gergelim

(Sesamum indicum L.) através da aplicação de regressão linear. E-xacta, v. 3, n. 1, p. 20-28, 2010.

CANCIAM, C. A. Aplicação da análise de regressão linear na predição do coeficiente de expansão térmica dos butiratos de etila, butila, isoamila e hexila. Revista da Universidade Vale do Rio Verde, v. 10, n. 2, p. 65-72, 2012a.

CANCIAM, C. A. Predição do coeficiente de expansão térmica dos ésteres: propionato de etila, isovalerato de etila e hexanoato de etila. Ciatec - UPF, v. 4, n. 1, p. 11-18, 2012 b.

CHUNG, S. K.; THIESSEN, D. B.; RHIM, D. B. A non contact measurement technique for the density and the thermal expansion coefficient of solids and liquid material. Review of Scientific Instruments, v. 67, n. 6, p. 3003-3381, 1996.

DJOJOPUTRO, H.; ISMADJI, S. Density and viscosity correlation for several common fragrance and flavor ester. Journal of Chemical \& Engineering Data, v. 50, n. 2, p. 727-731, 2005.

INCROPERA, F. P.; DEWITT, D. P. Fundamentos de transferência de calor e massa. Livros Técnicos e Científicos, Rio de Janeiro, 1998. 494p.

LIRA, S. A. Análise de correlação: abordagem teórica e de construção dos coeficientes com aplicação. Dissertação de Mestrado do Programa de Mestrado em Ciências da Universidade Federal do Paraná, 2004. 196p.
PADILHA, A. F. Materiais de Engenharia: microestrutura e propriedades. Hemus, São Paulo, 1997. 349p.

SANTOS, D. Q.; VIEIRA, D. F. Determinação de coeficiente de expansão térmica do biodiesel e seus impactos no sistema de medição volumétrico. Eclética Química, v. 35, n. 4, p. 107-112, 2010.

SKORONSKI, E. Estudo cinético da síntese do octanoato de n-pentila catalisada pela enzima Lipozyme TL IM. Dissertação de Mestrado do Programa de Mestrado em Engenharia Química da Universidade Federal de Santa Catarina, 2006. 87p.

SOLOMONS, T. W. G.; FRYHLE, C. B. Química Orgânica - volume 2. LTC, Rio de Janeiro, 2012. 644p.

TRIOLA, M. F. Introdução a Estatística. Livros Técnicos e Científicos, Rio de Janeiro, 2008. 410p.

VIEIRA, M. A. R. Caracterização dos ácidos graxos das sementes e compostos voláteis dos frutos de espécies do gênero Passiflora. Dissertação de Mestrado do Programa de Mestrado em Agronomia da Universidade Estadual "Júlio de Mesquita Filho", 2006. $71 \mathrm{p}$. 